\title{
Prospects for neutrino astrophysics with Hyper-Kamiokande
}

\section{Takatomi Yano* on behalf of the Hyper-Kamiokande Collaboration}

(a complete list of authors can be found at the end of the proceedings)

Kamioka Observatory, ICRR, The University of Tokyo, 456 Mozumi, Kamioka, Hida, Gifu, Japan

E-mail: tyano@km.icrr.u-tokyo.ac.jp

Hyper-Kamiokande is a multi-purpose next generation neutrino experiment. The detector is a two-layered cylindrical shape ultra-pure water tank, with its height of $64 \mathrm{~m}$ and diameter of 71 $\mathrm{m}$. The inner detector will be surrounded by tens of thousands of twenty-inch photosensors and multi-PMT modules to detect water Cherenkov radiation due to the charged particles and provide our fiducial volume of $188 \mathrm{kt}$. This detection technique is established by Kamiokande and Super-Kamiokande. As the successor of these experiments, Hyper-K will be located deep underground, $600 \mathrm{~m}$ below Mt. Tochibora at Kamioka in Japan to reduce cosmic-ray backgrounds. Besides our physics program with accelerator neutrino, atmospheric neutrino and proton decay, neutrino astrophysics is an important research topic for Hyper-K. With its fruitful physics research programs, Hyper-K will play a critical role in the next neutrino physics frontier. It will also provide important information via astrophysical neutrino measurements, i.e., solar neutrino, supernova burst neutrinos and supernova relic neutrino. Here, we will discuss the physics potential of HyperK neutrino astrophysics.

$37^{\text {th }}$ International Cosmic Ray Conference (ICRC 2021)

July 12th - 23rd, 2021

Online - Berlin, Germany

\footnotetext{
${ }^{*}$ Presenter
} 


\section{Introduction}

Hyper-Kamiokande (Hyper-K, HK) is a next generation water Cherenkov detector planned in Japan [1-3], as a successor of the Super-Kamiokande (Super-K, SK) experiment [4]. With the dimensions of the $71 \mathrm{~m}(\mathrm{D}) \times 60 \mathrm{~m}(\mathrm{H})$, a cylindrical water tank will provide the fiducial (total) volume of $0.188(0.258)$ million metric tons (figure 1). They are 8 (5) times larger than those of Super-K. The target date for starting the operation is 2027 . The inner detector will be surrounded by 20 -inch diameter photodetectors and multi-PMT modules. The photo-coverage of $40 \%$ is aimed to achieve, which corresponds to 40,000 of 20-inch PMTs. Here, we also discuss about an option to install 20,000 of 20-inch PMTs and thousands of multi-PMT modules. Thousands of 3-inch diameter photodetectors with wavelength shifting plates will be provided for the outer veto detector, to remove cosmic-ray muon backgrounds. The 20 -inch photodetector, Box\&Line dynode photo-multiplier tube Hamamatsu R12860, is newly developed for HK, to achieve twice larger detection efficiency for Cherenkov photons, the superior photon counting and timing resolution compared to that of SK (Hamamatsu R3600) [5]. It also has the high pressure tolerance for the usage below $60 \mathrm{~m}$ depth of water. The multiple-photosensor unit is also in our R\&D for the detector [5, 6]. The detector will be located underground at Kamioka mine in Gifu Prefecture, with an overburden of $\sim 600$ meters or more of rock, which is equivalent to 1,620 meters or more of water. Charged particles, such as the products of neutrino interactions, are detected with the emitted Cherenkov photons. The number of photons and their arrival times on the photodetectors are used to reconstruct the energy and vertex of the particle, respectively. Hyper-K has various physics topics: search for $\mathrm{CP}$ violation in neutrinos, precise study of neutrino oscillations including determination of mass hierarchy and $\theta_{23}$ octant with beam and atmospheric neutrinos, search for nucleon decay and observations of astrophysical neutrinos.

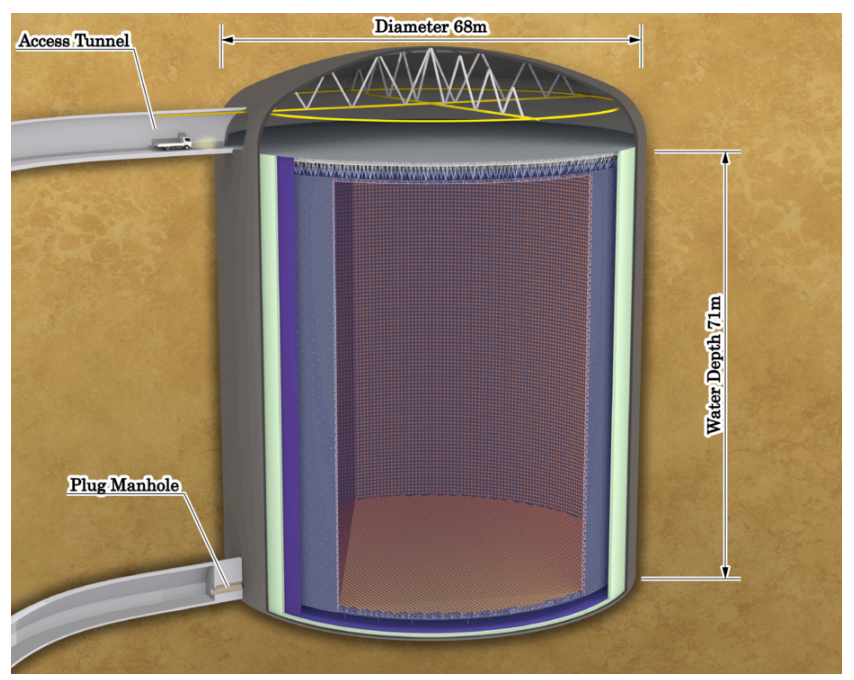

Figure 1: Schematic view of one Hyper-Kamiokande water Cherenkov detector [1]. Our collaboration aims at starting the observations at 2027. The tank will provide the fiducial volume of $0.188 \mathrm{Mt}$ ultra pure water, with the dimensions of the $71 \mathrm{~m}(\mathrm{D}) \times 60 \mathrm{~m}(\mathrm{H})$. 


\section{Solar Neutrino}

The Sun is burning and emitting neutrinos with the nuclear fusion reactions, which are called as the pp-chain and the $\mathrm{CNO}$ cycle. They can be summarized as follows: $4 p \rightarrow \alpha+2 e^{+}+2 v_{e}$. These processes are described with the standard solar model (SSM) [7, 8], which provides good predictions of the flux and energy spectrum of solar neutrinos. Our main observation target is the ${ }^{8} \mathrm{~B}$ neutrino, with its energy above our analysis threshold of $\mathrm{E}_{v i s}>4.5 \mathrm{MeV}$. Here, $\mathrm{E}_{v i s}$ is the visible energy of the neutrino event in water Cherenkov detector. It is smaller than the total energy of neutrinos by $\sim 1.5 \mathrm{MeV}$. They are observed through neutrino-electron elastic scattering, $v+e \rightarrow v+e$. The energy, direction, and time of the original neutrinos are measured through their recoil electrons. About $130 v$-e scattering events will be observed in a day, while $15 v$ events/day are observed at SK-I.

\subsection{Solar Neutrino Oscillation}

The solar neutrino measurement are capable of determining the neutrino oscillation parameters between neutrino mass eigenstates. Super-K [9], SNO [10] and several experiments [11-13] have been performed the neutrino oscillation measurement on the solar neutrinos. Figure 2 shows the latest results [14] of the allowed neutrino oscillation parameters, the mixing angle $\theta_{12}$ and the mass squared difference $\Delta m_{21}^{2}$ from all solar neutrino experiments, as well as the reactor neutrino experiment KamLAND [15]. The $\Delta m_{21}^{2}$ of $6.11_{-0.68}^{+1.21} \times 10^{-5} \mathrm{eV}^{2}$ is reported there by SuperKamiokande collaboration, as the results of solar oscillation analysis combining Super-K and SNO measurements. The tension between solar and reactor best fit $\Delta m_{21}^{2}$ became rather small as $\sim 1.4 \sigma$,

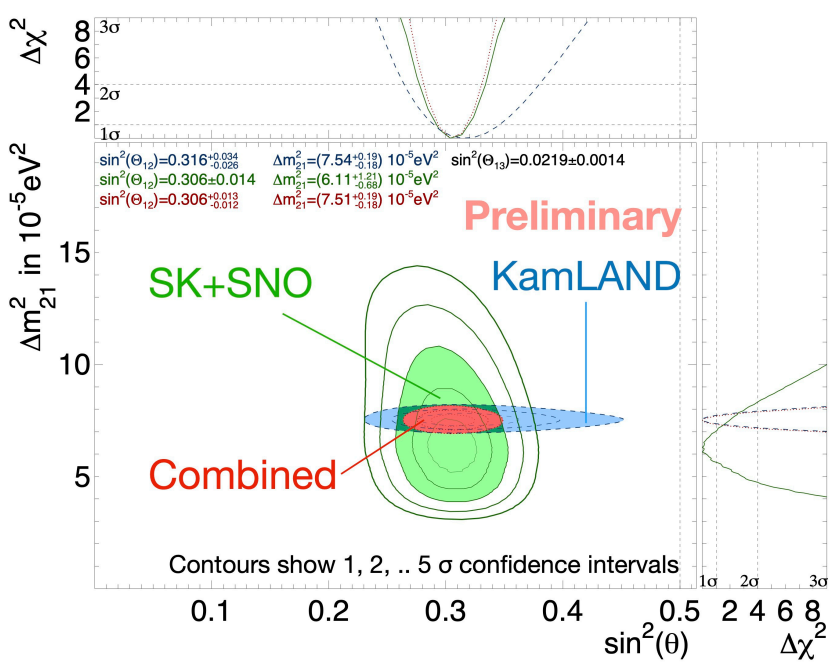

Figure 2: Neutrino oscillation parameter allowed region from all the solar experiments (green), KamLAND (blue) and Solar+KamLAND (red) from 1 to $5 \sigma$ lines and $3 \sigma$ area are shown[14]. The dashed green line is the combined results of SK and SNO.

comparing to $\sim 2 \sigma$ tension with solar best fit $\Delta m_{21}^{2}=4.8_{-0.8}^{+1.5} \times 10^{-5} \mathrm{eV}^{2}$ in 2016 [9].

The tension is mainly derived from the asymmetry of the solar neutrino flux during day and night (day-night asymmetry), which was indicated by Super-K [16]. The asymmetry would arises 
from the terrestrial matter effect, i.e. the regeneration of the electron neutrinos through MSW matter effect in the Earth. The effect can be seen as a few percent more event rate in the nighttime, than that in the daytime. With Hyper-K, the day-night asymmetry effect can be measured precisely with our large detector volume. Assuming the solar best fit $\Delta m_{21}^{2}$ parameter at 2020, our measurement will be possible to prove the day-night asymmetry effect above $5 \sigma$ (figure 3 ) with 10 years observation.

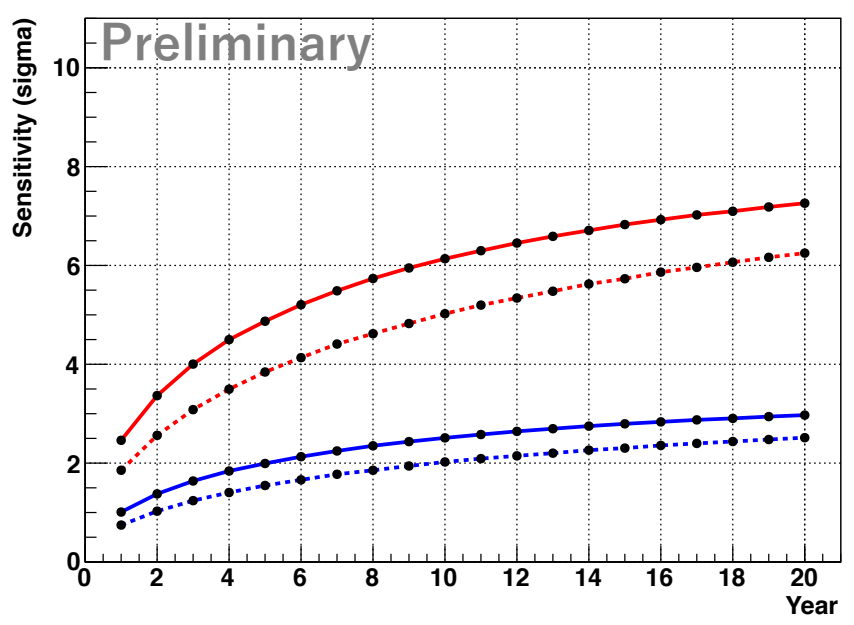

Figure 3: Day-night asymmetry observation sensitivity as a function of observation time. The red line shows the sensitivity from the no asymmetry, while the blue line shows from the asymmetry expected by the reactor neutrino oscillation. The solid line is for Hyper-K inner detector photo-coverage of $40 \%$, while the dotted line is for $20 \%$ case. The systematic uncertainty of $0.3 \%$ is assumed here.

The solar neutrino survival probability upturn is also the interesting physics properties. It is predicted by MSW-LMA solution and possibly affected by physics beyond the standard model, such as non-standard interaction[17], mass-varying neutrino oscillation[18] and sterile neutrino[19], for example. The non-zero upturn sensitivity will be about $3 \sigma(4 \sigma)$ after the 10 years solar neutrino measurement with $4.5 \mathrm{MeV}(3.5 \mathrm{MeV})$ threshold, even with the solar best fit oscillation parameters in 2020 .

\section{Supernova Neutrinos}

Core collapse supernova explosions are the last process in the evolution of massive stars $(>8$ $\mathrm{M}_{\odot}$ ). The energy released by a supernova is estimated to be $\sim 3 \times 10^{53}$ ergs and $99 \%$ of the energy is carried out by all three types of neutrinos and anti-neutrinos. The detection of supernova neutrinos gives direct information of energy flow during the explosions. From SN1987a, the Kamiokande, IMB, and Baksan experiments observed 25 neutrino events. It proved the basic scenario of the supernova explosion was correct. However, close to three decades later the detailed mechanism of explosions is still not known. The observation of new supernova with the large neutrino detector is desired. The multi-messenger observation with visible light, gamma-ray, $\mathrm{x}$-ray, gravitational wave and Hyper-K will also reveal the supernova explosion in details. 


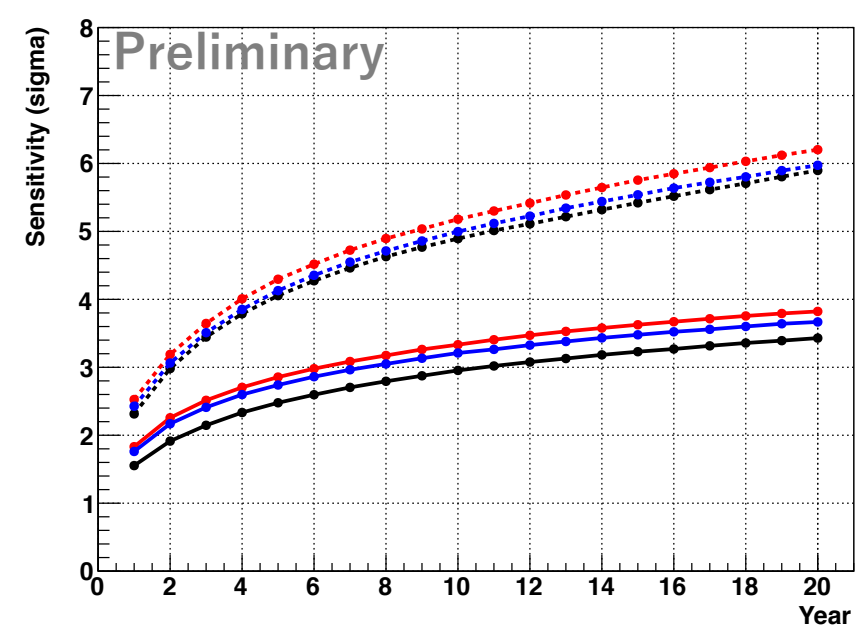

Figure 4: Discovery sensitivity for solar neutrino spectrum upturn as a function of the observation time. The solid line shows the case with the energy threshold of $4.5 \mathrm{MeV}$, while the dotted line shows the case with the energy threshold of $3.5 \mathrm{MeV}$. Black line shows the case with photo-coverage of $40 \%$, solar best fit $\Delta m_{21}^{2}$ and $\theta_{12}$ in 2016. The red (blue) line shows the updated sensitivity for photo-coverage of $40 \%(20 \%)$ and solar best fit parameters in 2020 .

The first and direct observation of supernova neutrinos is about the supernova burst neutrinos, which are released in several seconds after its onset of a burst. About $90 \%$ of signals at Hyper-K is inverse beta decay reaction $\left(\bar{v}_{e}+p \rightarrow e^{+}+n\right)$. For each full volume of two inner detectors, we expect to see about 49,000-68,000 inverse beta decay events, 2,100-2,500 v-e scattering events, $80-4,100 v_{e}+{ }^{16} \mathrm{O} \mathrm{CC}$ events, and 650-3,900 $\overline{v_{e}}+{ }^{16} \mathrm{O} \mathrm{CC}$ events, in total 52,000-79,000 events for a supernova explosion at halfway across our galaxy $(10 \mathrm{kpc}$, figure 5). The statistical error will be small enough to compare several SN models, and so Hyper-K should give crucial data for further model predictions (figure 6). A new characteristic modulation of the supernova neutrino flux also can be tested with HK, which the recent computer simulations predict. In past simulation, the deceleration of the shock wave and resulting failed explosion were annoying problems. Recent simulations suggest that the shock wave will be heated efficiently by neutrinos to revival, due to the physical motions in a supernova, Standing Accretion Shock Instability (SASI) or convection, rotation of supernova are the examples. These models also predict the characteristic frequency modulation of neutrino flux, due to the motions in supernovae. The detection of these modulation will prove the neutrino as the driver of supernova explosions. Other topics for astrophysics and particle physics also can be examined, e.g. direct observation of black hole formations and mass hierarchy of neutrinos.

Another observation target is about the supernova relic neutrinos (SRN), produced by all past supernova explosions since the beginning of the universe and diffused. They must fill the universe and their flux is estimated to be a few tens $/ \mathrm{cm}^{2} / \mathrm{sec}$. SRN contains the information of its origins, i.e. the star formation rate, energy spectrum of supernova burst neutrinos, and the fraction of strange supernova explosions like dim supernovae or black hole formations (figure 7). Although searches for SRN have been conducted at large underground detectors, no evidence of SRN signals has yet been obtained, because of the small flux of SRN. With incoming detector update, Gd-loaded 


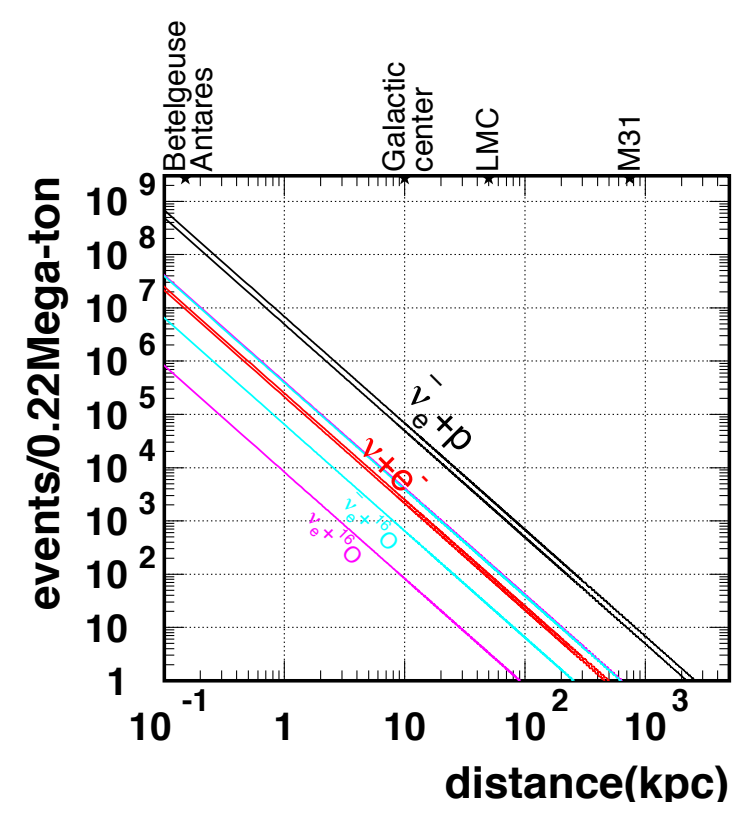

Figure 5: Expected number of supernova burst events for each interaction as a function of the distance to a supernova[3]. The band of each line shows the possible variation due to the assumption of neutrino oscillations.

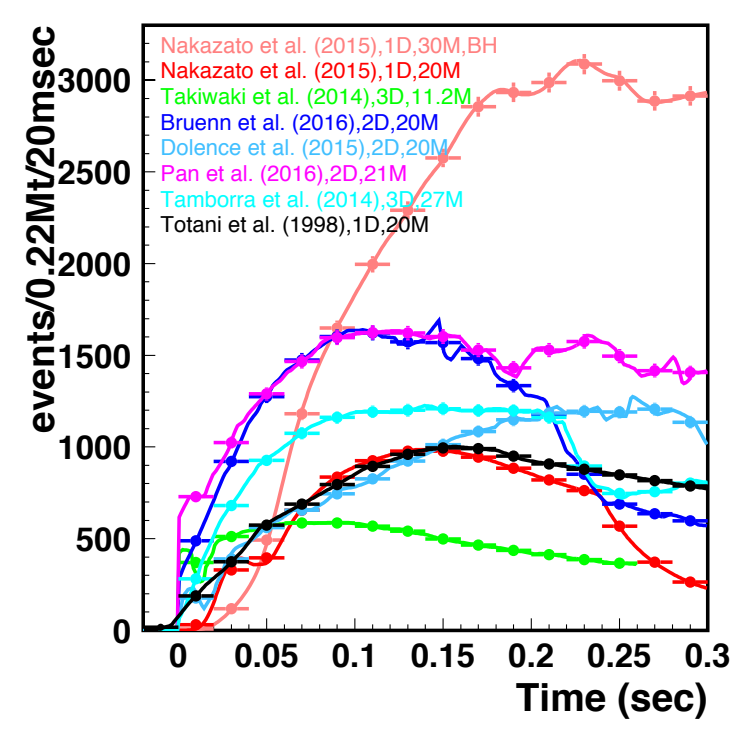

Figure 6: Inverse beta decay event rate predicted by supernova simulations for the first 0.3 seconds after the onset of a $10 \mathrm{kpc}$ distant burst[3]. The error bars show the statistical error.

Super-Kamiokande (SK-Gd) can be the discoverer of SRN. The number of event in SK-Gd detector will be 0.8-5 events/year above $10 \mathrm{MeV}$. Even though, it is still very interesting physics theme to measure and determine the precise flux of SRN. 70 (40) SRN events are expected at 16-30 MeV by 10 years observation with the detector photo-coverage of $40 \%(20 \%)$. The significance will be $4.2 \sigma$ and enough for confirming the discovery (figure 8). The significance will be $3 \sigma$, in case of photo-coverage of $20 \%$.

\section{Summary}

Hyper-Kamiokande is a next generation large water Cherenkov detector. Several studies are being performed, e.g. photosensor $\mathrm{R} \& \mathrm{D}$, design and physics optimization. Solar neutrino measurement is one of the features of Hyper-K. Several precise measurements of solar neutrinos would be possible with Hyper-K and its high statistics, e.g. the solar neutrino oscillation, the search for physics beyond the standard model, the first measurement of hep process neutrino and also the seasonal variation measurement of the ${ }^{8} \mathrm{~B}$ neutrino flux. As a conclusion, Hyper-K will play a crucial role in the next neutrino physics frontier for both of particle physics and neutrino astrophysics.

\section{References}

[1] K. Abe et al., Letter of Intent: The Hyper-Kamiokande Experiment - Detector Design and Physics Potential -, 1109.3262. 


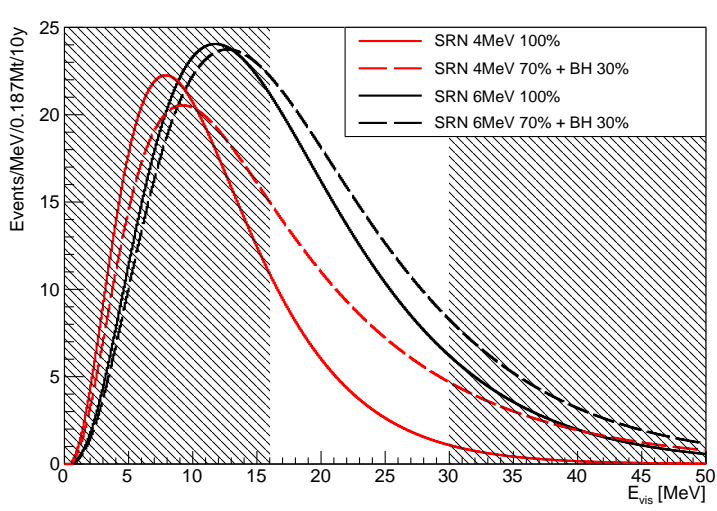

Figure 7: The expected SRN signal in 10 years observation is shown. Black line shows the case of supernova neutrino temperature of $6 \mathrm{MeV}$, and red shows the case of $4 \mathrm{MeV}$ [20,21]. Solid (dashed) line corresponds to the case, in which all the core-collapse supernovae emit neutrinos with the particular energy (30\% of them form black hole and emit higher energy neutrinos with the temperature of $8 \mathrm{MeV}$ ). Shaded energy region shows the range out of SRN search window at Hyper-K.

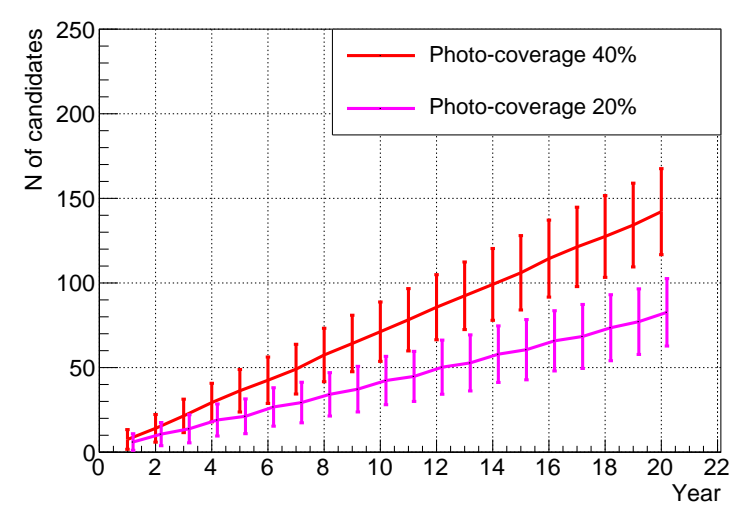

Figure 8: The figure shows the expected number of observed SRN events and its ambiguity as a function of observation period.

[2] Hyper-Kamiokande Working Group collaboration, A Long Baseline Neutrino Oscillation Experiment Using J-PARC Neutrino Beam and Hyper-Kamiokande, 2014, 1412.4673.

[3] Hyper-Kamiokande collaboration, Hyper-Kamiokande Design Report, 1805. 04163.

[4] Super-Kamiokande collaboration, The Super-Kamiokande detector, Nucl. Instrum. Meth. A501 (2003) 418.

[5] Hyper-Kamiokande Proto collaboration, New $50 \mathrm{~cm}$ Photo-Detectors for Hyper-Kamiokande, PoS ICHEP2016 (2017) 303.

[6] Hyper-Kamiokande proto collaboration, $R \& D$ Studies of New Photosensors for the Hyper-Kamiokande Detector, Springer Proc. Phys. 213 (2018) 325.

[7] J. N. Bahcall and M. H. Pinsonneault, What do we (not) know theoretically about solar neutrino fluxes?, Phys. Rev. Lett. 92 (2004) 121301 [astro-ph/0402114].

[8] J. N. Bahcall, A. M. Serenelli and S. Basu, 10,000 standard solar models: a Monte Carlo simulation, Astrophys. J. Suppl. 165 (2006) 400 [astro-ph/0511337].

[9] Super-Kamiokande collaboration, Solar Neutrino Measurements in Super-Kamiokande-IV, Phys. Rev. D94 (2016) 052010 [1606.07538].

[10] SNO collaboration, Combined Analysis of all Three Phases of Solar Neutrino Data from the Sudbury Neutrino Observatory, Phys. Rev. C88 (2013) 025501 [1109. 0763]. 
[11] B. T. Cleveland, T. Daily, R. Davis, Jr., J. R. Distel, K. Lande, C. K. Lee et al., Measurement of the solar electron neutrino flux with the Homestake chlorine detector, Astrophys. J. 496 (1998) 505.

[12] SAGE collaboration, Measurement of the solar neutrino capture rate with gallium metal. III: Results for the 2002-2007 data-taking period, Phys. Rev. C80 (2009) 015807 [0901 . 2200].

[13] G. Bellini et al., Precision measurement of the 7Be solar neutrino interaction rate in Borexino, Phys. Rev. Lett. 107 (2011) 141302 [1104. 1816].

[14] Y. Nakajima, Recent results and future prospects from Super- Kamiokande, June, 2020. 10.5281/zenodo.3959640.

[15] KamLAND collaboration, Reactor On-Off Antineutrino Measurement with KamLAND, Phys. Rev. D 88 (2013) 033001 [1303.4667].

[16] Super-Kamiokande collaboration, First Indication of Terrestrial Matter Effects on Solar Neutrino Oscillation, Phys. Rev. Lett. 112 (2014) 091805 [1312 . 5176].

[17] A. Friedland, C. Lunardini and C. Pena-Garay, Solar neutrinos as probes of neutrino matter interactions, Phys. Lett. B594 (2004) 347 [hep-ph/0402266].

[18] V. Barger, P. Huber and D. Marfatia, Solar mass-varying neutrino oscillations, Phys. Rev. Lett. 95 (2005) 211802 [hep-ph/0502196].

[19] P. C. de Holanda and A. Yu. Smirnov, Homestake result, sterile neutrinos and low-energy solar neutrino experiments, Phys. Rev. D69 (2004) 113002 [hep-ph/0307266].

[20] S. Horiuchi, J. F. Beacom and E. Dwek, The Diffuse Supernova Neutrino Background is detectable in Super-Kamiokande, Phys. Rev. D79 (2009) 083013 [0812 . 3157].

[21] S. Horiuchi, Private communication, Private communication (2017). 


\section{Full Authors List: Hyper-Kamiokande Collaboration}

K. Abe ${ }^{1,2,3}$, P. Adrich ${ }^{4}$, H. Aihara ${ }^{5,2,3}$, R. Akutsu ${ }^{6}$, I. Alekseev ${ }^{7}$, A. Ali ${ }^{8}$, Y. I. Alj Hakim ${ }^{9}$, F. Ameli ${ }^{10}$, L.H.V. Anthony ${ }^{9}$, A. Araya ${ }^{11,3}$, Y. Asaoka ${ }^{1,3}$, V. Aushev ${ }^{12}$, F. Ballester ${ }^{13}$, I. Bandac ${ }^{14}$, M. Barbi ${ }^{15}$, G. Barr ${ }^{16}$, M. Batkiewicz-Kwasniak ${ }^{17}$, M. Bellato ${ }^{18}$, V. Berardi ${ }^{19}$, L. Bernard ${ }^{20}$, E. Bernardini ${ }^{18}$, L. Berns ${ }^{21}$, S. Bhadra ${ }^{22}$, J. Bian ${ }^{23}$, A. Blanchet ${ }^{24}$, A. Blondel ${ }^{24}$, A. Boiano ${ }^{25}$, S. Bolognesi ${ }^{26}$, L. Bonavera ${ }^{27}$, N. Booth ${ }^{28}$, S. Bordoni ${ }^{29}$, S. Borjabad ${ }^{14}$, T. Boschi ${ }^{30}$, D. Bose ${ }^{31}$, S .B. Boyd ${ }^{32}$, C. Bozza ${ }^{33}$, A. Bravar ${ }^{29}$, C. Bronner ${ }^{1,3}$, L. Brown ${ }^{28}$, A. Bubak ${ }^{34}$, A. Buchowicz ${ }^{35}$, M. Buizza Avanzini ${ }^{20}$, F. S. Cafagna ${ }^{19}$, N. F. Calabria ${ }^{36}$, J. M. Calvo-Mozota ${ }^{14}$, S. Cao ${ }^{37,38}$, A. Carroll ${ }^{39}$, M. G. Catanesi ${ }^{19}$, S. Cebriàn ${ }^{40}$, S. Chakraborty ${ }^{41}$, J. H. Choir ${ }^{42}$, S. Choubey ${ }^{43}$, M. Cicerchia ${ }^{44}$, J. Coleman ${ }^{39}$, G. Collazuol ${ }^{18}$, S. Cuen-Rochin ${ }^{45,6}$, M. Danilov ${ }^{7}$, G. Díaz López ${ }^{46}$, E. De la Fuente ${ }^{47,48,49}$, P. de Perio ${ }^{6}$, G. De Rosa ${ }^{36}$, T. Dealtry ${ }^{50}$, C. J. Densham ${ }^{51}$, A. Dergacheva ${ }^{52}$, N. Deshmukh ${ }^{53}$, M. M. Devi ${ }^{54}$, F. Di Lodovico ${ }^{30}$, P. Di Meo ${ }^{25}$, I. Di Palma ${ }^{10}$, T. A. Doyle ${ }^{50}$, E. Drakopoulou ${ }^{55}$, O. Drapier ${ }^{20}$, J. Dumarchez ${ }^{24}$, L. Eklund ${ }^{56}$, S. El Hedri ${ }^{20}$, J. Ellis ${ }^{30}$, S. Emery ${ }^{26}$, A. Esmaili ${ }^{57}$, R. Esteve ${ }^{13}$, S. Fedotov ${ }^{52}$, J. Feng ${ }^{8}$, P. Fernandez ${ }^{39}$, E. Fernández-Martinez ${ }^{58}$, P. Ferrario ${ }^{59}$, B. Ferrazzi ${ }^{15}$, A. Finch ${ }^{50}$, C. Finley ${ }^{60}$, G. Fiorillo ${ }^{36}$, M. Fitton ${ }^{51}$, M. Friend ${ }^{37,38}$, Y. Fujii ${ }^{37,38}$, C. Fujisawa ${ }^{61}$, Y. Fukuda ${ }^{62}$, G. Galinski ${ }^{35}$, J. Gao $^{30}$, C. Garde ${ }^{53}$, A. Garfagnini ${ }^{18}$, S. Garode ${ }^{53}$, L. Gialanella ${ }^{63}$, C. Giganti ${ }^{24}$, J. J. Gomez-Cadenas ${ }^{59}$, M. Gonin ${ }^{20}$, J. González-Nuevo ${ }^{27}$, A. Gorin ${ }^{52}$, R. Gornea ${ }^{64}$, V. Gousy-Leblanc ${ }^{28}$, F. Gramegna ${ }^{44}$, M. Grassi ${ }^{18}$, G. Grella ${ }^{33}$, M. Guigue ${ }^{24}$, D. R. Hadley ${ }^{32}$, M. Harada ${ }^{65}$, M. Hartz ${ }^{2,6,3}$, S. Hassani ${ }^{26}$, N. C. Hastings ${ }^{37,38}$,

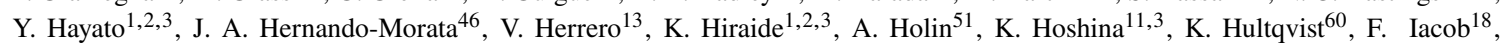
A. K. Ichikawa ${ }^{66}$, W. Idrissi Ibnsalih ${ }^{63}$, K. Ieki ${ }^{1}$, M. Ikeda ${ }^{1,2,3}$, A. Ioannisian ${ }^{67}$, T. Ishida ${ }^{37,38}$, K. Ishidoshiro ${ }^{68}$, H. Ishino ${ }^{65}$, M. Ishitsuka ${ }^{69}$, H. T. Israel ${ }^{70}$, H. Ito ${ }^{1}$, Y. Itow ${ }^{71,72}$, N. Izumi ${ }^{69}$, S. Izumiyama ${ }^{21}$, M. Jakkapu ${ }^{37,73}$, B. Jamieson ${ }^{74}$, J. S. Jang ${ }^{75}$, H. S. Jo ${ }^{76}$, P. Jonsson ${ }^{9}$, K. K. Joo ${ }^{77}$, T. Kajita ${ }^{49,2,3}$, H. Kakuno ${ }^{78}$, J. Kameda ${ }^{1,2,3}$, R. Kaneshima ${ }^{1}$, Y. Kano ${ }^{11,3}$, D. Karlen ${ }^{28,6}$, Y. Kataoka ${ }^{1,3}$, A. Kato ${ }^{11,3}$, T. Katori ${ }^{30}$, N. Kazarian ${ }^{67}$, M. Khabibullin' ${ }^{52}$, A. Khotjantsev ${ }^{52}$, T. Kikawa ${ }^{8}$, M. Kekic ${ }^{46}$, J. Y. Kim ${ }^{77}$, S. B. Kim $^{79}$, S. King ${ }^{30}$, T. Kinoshita ${ }^{69}$, J. Kisiel ${ }^{17,34}$, A. Klekotko ${ }^{35}$, T. Kobayashi ${ }^{37,38}$, L. Koerich ${ }^{15}$, N. Kolev ${ }^{15}$, A. Konaka ${ }^{6}$, L. L. Kormos ${ }^{50}$, Y. Koshio ${ }^{65,2}$, Y. Kotsar ${ }^{80}$, K. A. Kouzakov ${ }^{81}$, K.L. Kowalik ${ }^{4}$, L. Kravchuk ${ }^{52}$, A. P. Kryukov ${ }^{81}$, Y. Kudenko ${ }^{52}$,

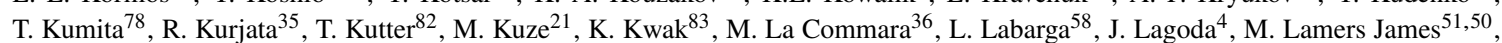

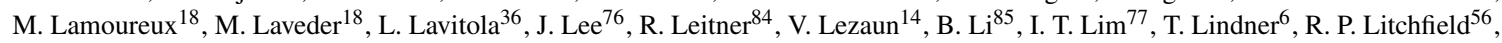
K. R. Long ${ }^{9}$, A. Longhin ${ }^{18}$, P. Loverre ${ }^{10}$, X. Lu ${ }^{16}$, L. Ludovici ${ }^{10}$, Y. Maekawa ${ }^{61}$, L. Magaletti ${ }^{19}$, K. Magar ${ }^{53}$, Y. Makida ${ }^{37,38}$, M. Malek ${ }^{70}$, M. Malinský ${ }^{84}$, T. Marchi ${ }^{44}$, C. Mariani ${ }^{86}$, A. Marinelli ${ }^{25}$, K. Martens ${ }^{2,3}$, Ll. Marti ${ }^{1,3}$, J. F. Martin ${ }^{87}$, D. Martin ${ }^{9}$, J. Marzec ${ }^{35}$, T. Matsubara ${ }^{37}, 38$, R. Matsumoto ${ }^{69}$, N. McCauley ${ }^{39}$, A. Medhi ${ }^{54}$, P. Mehta ${ }^{39}$, L. Mellet ${ }^{24}$, H. Menjo ${ }^{88}$, M. Mezzetto ${ }^{18}$, J. Migenda ${ }^{30}$, P. Migliozzi ${ }^{25}$, S. Miki ${ }^{1}$, E. W. Miller ${ }^{30}$, A. Minamino ${ }^{89}$, S. Mine $^{23}$, O. Mineev ${ }^{52}$, L. Munteanu ${ }^{26}$, A. Mitra ${ }^{32}$, M. Miura ${ }^{1,2,3}$, R. Moharana ${ }^{90}$, C. M. Mollo ${ }^{25}$, T. Mondal ${ }^{*}, 31$, M. Mongelli ${ }^{19}$, F. Monrabal ${ }^{59}$, D. H. Moon ${ }^{77}$, C. S. Moon ${ }^{76}$, F. J. Mora ${ }^{13}$, M. Mori ${ }^{8}$, S. Moriyama ${ }^{1,2,3}$, Th. A. Mueller ${ }^{20}$, T. Nakadaira ${ }^{37,38}$, K. Nakagiri ${ }^{5,3}$, M. Nakahata ${ }^{1,2,3}$, S. Nakai ${ }^{11,3}$, Y. Nakajima ${ }^{5,2,3}$, K. Nakamura ${ }^{37,2}$, KI. Nakamura ${ }^{66}$, H. Nakamura ${ }^{69}$, S. Nakanishi ${ }^{69}$, Y. Nakano ${ }^{80}$, T. Nakaya ${ }^{8,2}$, S. Nakayama ${ }^{1,2,3}$, K. Nakayoshi ${ }^{37,38}$, L. Nascimento Machado $^{36}$, C. E. R. Naseby ${ }^{9}$, B. Navarro-Garcia ${ }^{47}$, M. Needham ${ }^{55}$, K. Niewczas ${ }^{91}$, K. Ninomiya ${ }^{88}$, S. Nishimori ${ }^{37}$, Y. Nishimura ${ }^{61}$, Y. Noguchi ${ }^{8}$, F. Nova ${ }^{51}$, J. C. Nugent ${ }^{56}$, H. Nunokawa ${ }^{57}$, W. Obrebski ${ }^{35}$, J. P. Ochoa-Ricoux ${ }^{23}$, E. O'Connor ${ }^{92}$, N. Ogawa ${ }^{5}$, T. Ogitsu ${ }^{37,38}$, K. Okamoto ${ }^{1}$, H. M. O' Keeffe $^{50}$, K. Okumura $^{49,2,3}$, Y. Onishchuk ${ }^{12}$, F. Orozco-Luna ${ }^{48}$, A. Oshlianskyi ${ }^{12}$, N. Ospina ${ }^{18}$, M. Ostrowski ${ }^{93}$, E. O’Sullivan ${ }^{94}$, Y. Oyama ${ }^{37,38}$, H. Ozaki ${ }^{80}$, M.Y. Pac ${ }^{42}$, P. Paganini ${ }^{20}$, V. Palladino ${ }^{36}$, M. Pari ${ }^{18}$, J. Pasternak ${ }^{9}$, C. Pastore ${ }^{19}$, G. Pastuszak ${ }^{35}$, D. A. Patel ${ }^{15}$, M. Pavin ${ }^{6}$, D. Payne ${ }^{39}$, C. Peña-Garay ${ }^{14}$, C. Pidcott ${ }^{70}$, S. Playfer ${ }^{55}$, B. W. Pointon ${ }^{95,6}$, A. Popov ${ }^{81}$, B. Popov ${ }^{24}$, K. Porwit ${ }^{34}$, M. Posiadala-Zezula ${ }^{96}$, G. Pronost ${ }^{1,3}$, N.W. Prouse ${ }^{6}$, B. Quilain ${ }^{20}$, A. A. Quiroga ${ }^{57}$, E. Radicioni ${ }^{19}$, B. Radics ${ }^{85}$, P. J. Rajda ${ }^{97}$, J. Renner ${ }^{46}$, M. Rescigno ${ }^{10}$, G. Ricciardi ${ }^{36}$, B. Richards ${ }^{32}$, E. Rondio ${ }^{4}$, B. Roskovec ${ }^{84}$, S. Roth ${ }^{98}$, C. Rott ${ }^{79}$, A. Rubbia ${ }^{85}$, A.C. Ruggeri ${ }^{25}$, S. Russo ${ }^{24}$, A. Rychter ${ }^{35}$, D. Ryu ${ }^{83}$, K. Sakashita ${ }^{37,38}$, S. Samani ${ }^{16}$, F. Sánchez ${ }^{29}$, M. L. Sánchez ${ }^{27}$, S. Sano ${ }^{89}$, J. D. Santos ${ }^{27}$, G. Santucci ${ }^{22}$, P. Sarmah ${ }^{41}$, K. Sato ${ }^{88}$, M. Scott ${ }^{9}$, Y. Seiya ${ }^{99}$, T. Sekiguchi ${ }^{37,38}$, H. Sekiya ${ }^{1,2,3}$, J. W. Seo ${ }^{79}$, D. Sgalaberna ${ }^{85}$, Z. Shan ${ }^{30}$, A. Shaykina ${ }^{52}$, H. Shiba ${ }^{1}$, I. Shimizu ${ }^{68}$, K. Shimizu ${ }^{1}$, C. D. Shin ${ }^{77}$, M. Shinoki ${ }^{69}$, M. Shiozawa ${ }^{1,2,3}$, N. Skrobova ${ }^{7}$, K. Skwarczynski ${ }^{4}$, M.B. Smy ${ }^{23,2}$, J. Sobczyk ${ }^{91}$, H. W. Sobel ${ }^{23,2}$, F. J. P. Soler ${ }^{56}$, Y. Sonoda ${ }^{1}$, R. Spina ${ }^{19}$, B. Spisso ${ }^{100}$, P. Spradlin ${ }^{56}$, K. L. Stankevich ${ }^{81}$, L. Stawarz ${ }^{33}$, S. M. Stellacci ${ }^{100}$, A. I. Studenikin ${ }^{81}$, S. L. Suárez Gómez ${ }^{27}$, T. Suganuma ${ }^{69}$, S. Suvorov ${ }^{52}$, Y. Suwa ${ }^{101}$, A. T. Suzuki ${ }^{80}$, S. Y. Suzuki ${ }^{37,38}$, Y. Suzuki ${ }^{102}$, D. Svirida ${ }^{7}$, M. Taani ${ }^{30}$, M. Tada $^{37,38}$, A. Takeda ${ }^{1,2,3}$, Y. Takemoto ${ }^{1,2,3}$, A. Taketa ${ }^{11,3}$, Y. Takeuchi ${ }^{80,2}$, H. Tanaka ${ }^{1,2,3}$, H. I. Tanaka ${ }^{11,3}$, M. Tanaka ${ }^{37,38}$, T. Tashiro ${ }^{49,3}$, M. Thiesse ${ }^{70}$, L. F. Thompson ${ }^{70}$, D. Tiwari ${ }^{15}$, J. F. Toledo ${ }^{13}$, A. K. Tomatani-Sánchez ${ }^{103}$, G. Tortone ${ }^{25}$, K. M. Tsui ${ }^{39}$, T. Tsukamoto ${ }^{37,38}$,

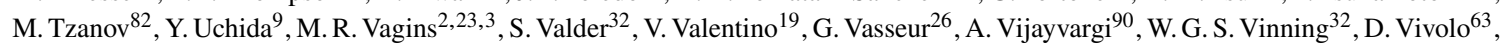
R. B. Vogelaar ${ }^{86}$, M. M. Vyalkov ${ }^{81}$, T. Wachala ${ }^{17}$, D. Wark ${ }^{16,51}$, M. O. Wascko ${ }^{9}$, R. A. Wendell ${ }^{8,2}$, J. R. Wilson ${ }^{30}$, S. Wronka $^{4}$, J. Xia $^{49}$,

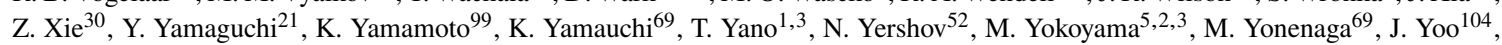
I. Yu ${ }^{79}$, T. Zakrzewski ${ }^{4}$, B. Zaldivar ${ }^{58}$, J. Zalipska ${ }^{4}$, K. Zaremba ${ }^{35}$, G. Zarnecki ${ }^{4}$, M. Ziembicki ${ }^{35}$, K. Zietara ${ }^{93}$, M. Zito ${ }^{24}$, T Zhu ${ }^{9}$ and S. Zsoldos ${ }^{30}$

${ }^{1}$ University of Tokyo, Institute for Cosmic Ray Research, Kamioka Observatory, Kamioka, Japan. ${ }^{2}$ University of Tokyo, Kavli Institute for the Physics and Mathematics of the Universe (WPI), University of Tokyo Institutes for Advanced Study, Kashiwa, Japan. ${ }^{3}$ University of Tokyo, Next-generation Neutrino Science Organization, Kamioka, Japan. ${ }^{4}$ National Centre for Nuclear Research, Warsaw, Poland. ${ }^{5}$ University of Tokyo, Department of Physics, Tokyo, Japan. ${ }^{6}$ TRIUMF, Vancouver, British Columbia, Canada. ${ }^{7}$ P.N.Lebedev Physical Institute of the Russian Academy of Sciences, Moscow, Russia. ${ }^{8}$ Kyoto University, Department of Physics, Kyoto, Japan. ${ }^{9}$ Imperial College London, Department of Physics, London, United Kingdom. ${ }^{10}$ INFN Sezione di Roma, Università Sapienza, Dipartimento di Fisica, Roma, Italy. ${ }^{11}$ University of Tokyo, Earthquake Research Institute, Tokyo, Japan. ${ }^{12}$ Kyiv National University, Department of Nuclear Physics, Kyiv, Ukraine. ${ }^{13}$ Universitat Politècnica de València, Instituto de Instrumentaciòn para Imagen Molecular (i3M), Valencia, Spain. ${ }^{14}$ Laboratorio Subterráneo de Canfranc, Canfranc-Estación, Spain. ${ }^{15}$ University of Regina, Department of Physics, Regina, Saskatchewan, Canada. ${ }^{16}$ Oxford University, Department of Physics, Oxford, United Kingdom. ${ }^{17} \mathrm{H}$. Niewodniczański Institute 
of Nuclear Physics PAN, Cracow, Poland. ${ }^{18}$ INFN Sezione di Padova and Università di Padova, Dipartimento di Fisica, Padova, Italy. ${ }^{19}$ INFN Sezione di Bari and Università e Politecnico di Bari,Bari Italy. ${ }^{20}$ Ecole Polytechnique, IN2P3-CNRS, Laboratoire LeprinceRinguet, Palaiseau, France. ${ }^{21}$ Tokyo Institute of Technology, Department of Physics, Tokyo, Japan. ${ }^{22}$ York University, Department of Physics and Astronomy, Toronto, Ontario, Canada. ${ }^{23}$ University of California, Irvine, Department of Physics and Astronomy, Irvine, California, USA. ${ }^{24}$ Laboratoire de Physique Nucleaire et de Hautes Energie, IN2P3/CNRS, Sorbonne Universitè, Paris, France. ${ }^{25}$ INFN Sezione di Napoli, Napoli, Italy. ${ }^{26}$ IRFU, CEA, Universitè Paris-Saclay, Gif-sur-Yvette, France. ${ }^{27}$ University of Oviedo, Applied Mathematical Modeling Group/Department of Physics, Oviedo, Spain. ${ }^{28}$ University of Victoria, Department of Physics and Astronomy, Victoria, British Columbia, Canada. ${ }^{29}$ University of Geneva, Section de Physique, DPNC, Geneva, Switzerland. ${ }^{30}$ King's College London, Department of Physics, Strand Building, Strand, London, United Kingdom. ${ }^{31}$ S. N. Bose National Centre for Basic Sciences, Salt Lake City, Kolkata, India. ${ }^{32}$ University of Warwick, Department of Physics, Coventry, United Kingdom. ${ }^{33}$ Università degli Studi di Salerno and INFN Gruppo Collegato di Salerno, Fisciano, Italy. ${ }^{34}$ University of Silesia in Katowice, A. Chełkowski Institute of Physics, Poland. ${ }^{35}$ Warsaw University of Technology, Institute of Radioelectronics and Multimedia Technology, Warsaw, Poland. ${ }^{36}$ INFN Sezione di Napoli and Università Federico II di Napoli, Dipartimento di Fisica, Napoli, Italy. ${ }^{37}$ High Energy Accelerator Research Organization (KEK), Tsukuba, Japan. ${ }^{38}$ J-PARC Center, Tokai, Japan. ${ }^{39}$ University of Liverpool, Department of Physics, Liverpool, United Kingdom. ${ }^{40}$ University of Zaragoza, Centro de Astropartículas y Física de Altas Energías (CAPA), Zaragoza, Spain. ${ }^{41}$ Indian Institute of Technology Guwahati, Guwahati, India. ${ }^{42}$ Dongshin University, Laboratory for High Energy Physics, Naju, Korea. ${ }^{43}$ KTH Royal Institute of Technology, Department of Physics, Stockholm, Sweden. ${ }^{44}$ INFN Laboratori Nazionali di Legnaro, Legnaro (PD), Italy. ${ }^{45}$ Universidad Autonoma de Sinaloa, Culiacan, Mexico. ${ }^{46}$ Universitat de Santiago de Compostela, Instituto Gallego de Física de Altas Enerxías, Santiago de Compostela, Spain. ${ }^{47}$ Universidad de Guadalajara, CUCEI, Departamento de Fisica, Guadalajara, Jal., Mexico. ${ }^{48}$ Universidad de Guadalajara, CUCEA, IT.Ph.D. program, Guadalajara, Jal., Mexico. ${ }^{49}$ University of Tokyo, Institute for Cosmic Ray Research, Research Center for Cosmic Neutrinos, Kashiwa, Japan. ${ }^{50}$ Lancaster University, Physics Department, Lancaster, United Kingdom. ${ }^{51}$ STFC, Rutherford Appleton Laboratory, Harwell Oxford, and Daresbury Laboratory, Warrington, United Kingdom. ${ }^{52}$ Institute for Nuclear Research of the Russian Academy of Sciences, Moscow, Russia. ${ }^{53}$ Vishwakarma Institute of Information Technology, Pune, India. ${ }^{54}$ Tezpur University, Department of Physics, Sonitpur, India. ${ }^{55}$ University of Edinburgh, School of Physics and Astronomy, Edinburgh, United Kingdom. ${ }^{56}$ University of Glasgow, School of Physics and Astronomy, Glasgow, United Kingdom. ${ }^{57}$ Pontifícia Universidade Católica do Rio de Janeiro, Departamento de Física, Rio de Janeiro, Brazil. ${ }^{58}$ University Autonoma Madrid, Department of Theoretical Physics, Madrid, Spain. ${ }^{59}$ Donostia International Physics Center and Ikerbasque Foundation, Basque Country, Spain. ${ }^{60}$ Stockholm University, Oskar Klein Centre and Department of Physics, Stockholm, Sweden. ${ }^{61}$ Keio University, Department of Physics, Yokohama, Japan. ${ }^{62}$ Miyagi University of Education, Department of Physics, Sendai, Japan. ${ }^{63}$ Università della Campania "L. Vanvitelli" and INFN Sezione di Napoli, Napoli, Italy. ${ }^{64}$ Carleton University, Department of Physics, Ottawa, Ontario, Canada. ${ }^{65}$ Okayama University, Department of Physics, Okayama, Japan. ${ }^{66}$ Tohoku University, Department of Physics, Sendai, Japan. ${ }^{67}$ Institute for Theoretical Physics and Modeling, Yerevan, Armenia. ${ }^{68}$ Tohoku University, Research Center for Neutrino Science, Sendai, Japan. ${ }^{69}$ Tokyo University of Science, Department of Physics, Chiba, Japan. ${ }^{70}$ University of Sheffield, Department of Physics and Astronomy, Sheffield, United Kingdom. ${ }^{71}$ Nagoya University, Kobayashi-Maskawa Institute for the Origin of Particles and the Universe, Nagoya, Japan. ${ }^{72}$ Nagoya University, Institute for Space-Earth Environmental Research, Nagoya, Japan. ${ }^{73}$ SOKENDAI (The Graduate University for Advanced Studies), Tokai, Japan. ${ }^{74}$ University of Winnipeg, Department of Physics, Winnipeg, Manitoba, Canada. ${ }^{75}$ GIST College, Gwangju Institute of Science and Technology, Gwangju, Korea. ${ }^{76}$ Kyungpook National University, Department of Physics, Daegu, Korea. ${ }^{77}$ Chonnam National University, Department of Physics, Gwangju, Korea. ${ }^{78}$ Tokyo Metropolitan University, Department of Physics, Tokyo, Japan. ${ }^{79}$ Sungkyunkwan University, Department of Physics, Suwon, Korea. ${ }^{80}$ Kobe University, Department of Physics, Kobe, Japan. ${ }^{81}$ Moscow State University, Department of Theoretical Physics, Moscow, Russia. ${ }^{82}$ Louisiana State University, Department of Physics and Astronomy, Baton Rouge, Louisiana, USA ${ }^{83}$ Ulsan National Institute of Science and Technology, Department of Physics, Ulsan, Korea. ${ }^{84}$ Charles University, IPNP, FMF, Prague, Czech. ${ }^{85}$ ETH Zurich, Institute for Particle and Astroparticle Physics, Zurich, Switzerland. ${ }^{86}$ Virginia Tech, Center for Neutrino Physics, Blacksburg, Virginia, USA. ${ }^{87}$ University of Toronto, Department of Physics, Toronto, Ontario, Canada. ${ }^{88}$ Nagoya University, Graduate School of Science, Nagoya, Japan. ${ }^{89}$ Yokohama National University, Faculty of Engineering, Yokohama, Japan. ${ }^{90}$ Indian Institute of Technology Jodhpur, Department of Physics, Karwar, Rajasthan, India. ${ }^{91}$ Wroclaw University, Faculty of Physics and Astronomy, Wroclaw, Poland. ${ }^{92}$ Stockholm University, Oskar Klein Centre and Department of Astronomy, Stockholm, Sweden. ${ }^{93}$ Astronomical Observatory of the Jagiellonian University, Krakow, Poland. ${ }^{94}$ Uppsala University, Department of Physics and Astronomy, Uppsala, Sweden. ${ }^{95}$ British Columbia Institute of Technology, Physics Department, Burnaby, British Columbia, Canada. ${ }^{96}$ University of Warsaw, Faculty of Physics, Warsaw, Poland. ${ }^{97}$ AGH University of Science and Technology, Faculty of Computer Science, Electronics and Telecommunications, Krakow, Poland. ${ }^{98}$ RWTH Aachen University, III. Physikalisches Institut, Aachen, Germany. ${ }^{99}$ Osaka City University, Department of Physics, Osaka, Japan. ${ }^{100}$ INFN Gruppo Collegato di Salerno, Fisciano, Italy. ${ }^{101}$ Kyoto Sangyo University, Department of Astrophysics and Atmospheric Sciences, Kyoto, Japan. ${ }^{102}$ University of Tokyo, Tokyo, Japan. ${ }^{103}$ Tecnologico de Monterrey, Escuela de Ingenieria y Ciencias, Zapopan, Jalisco, Mexico. ${ }^{104}$ Korea Institute of Science and Technology, Department of Physics, Daejeon, Korea.

${ }^{*}$ The current affiliation is Indian Institute of Technology Kharagpur, Department of Physics, Kharagpur, India. 